\title{
A ripping bike race: spontaneous coronary dissection complicated by cardiac tamponade, unmasking vascular Ehlers-Danlos in a young man
}

\author{
Inne Hendrickx, ${ }^{1}$ Benjamin Scott ${ }^{2}$ \\ ${ }^{1}$ Emergency Department, AZ Maria Middelares, Gent; ${ }^{2}$ Hartcentrum ZNA, ZNA Middelheim Hospital, \\ Antwerpen, Belgium
}

\begin{abstract}
Spontaneous coronary artery dissection (SCAD) is a rare cause of acute coronary syndrome. Typically, it occurs in young women without atherosclerotic risk factors. Clinical presentation ranges from chest pain to myocardial infarction, ventricular fibrillation and sudden death. We report a very rare case of a young man with ST-elevation myocardial infarction caused by SCAD, complicated by hemopericardium and recurrent cardiac tamponade. Due to this acute complication, he was diagnosed as having vascular EhlersDanlos syndrome.
\end{abstract}

\section{Case Report}

A 30-year-old male presented at the emergency department with severe upper-back and chest pain, which had started the day before. He had been cycling vigorously for several hours (the Tour of Flanders amateur race). He was hemodynamically stable with a blood pressure of $130 / 68 \mathrm{mmHg}$, heart rate of 100/min and saturation of $98 \%$ on room air. He had a medical history of spontaneous pneumothorax and a forearm fracture. Clinical examination revealed somewhat muffled heart tones but clear lung auscultation, normal abdominal investigation and normal peripheral pulsations. The ECG showed sinus tachycardia and deep T-wave inversion and

\footnotetext{
Correspondence: Benjamin Scott, Hartcentrum ZNA, ZNA Middelheim Hospital, Lindendreef 1, 2020 Antwerpen, Belgium.

E-mail: Benjamin.scott@zna.be

Key words: Spontaneous coronary artery dissection; Cardiac tamponade; Ehler-Danlos syndrome.

Contributions: the authors contributed equally.

Conflict of interest: the authors declare no potential conflict of interest.

Funding: none.

Received for publication: 4 September 2018.

Revision received: 10 February 2019.

Accepted for publication: 11 February 2019.

This work is licensed under a Creative Commons Attribution 4.0 License (by-nc 4.0).

(C) Copyright I. Hendrickx and B. Scott, 2019

Licensee PAGEPress, Italy

Emergency Care Journal 2019; 15:7802

doi:10.4081/ecj.2019.7802
}

mild ST-elevation in the lateral and inferior leads, consistent with ST- elevation myocardial infarction. Blood results showed elevated cardiac enzymes, consistent with subacute myocardial infarction. He underwent urgent coronary angiography, revealing an image suggestive of type 2 spontaneous dissection of large marginal branch of the circumflex coronary $\operatorname{artery}^{1}$ (Figure 1). Besides this, his coronary arteries were normal. Left ventricular angiography showed regional akinesia in the inferolateral wall. The patient was conservatively treated with aspirin and nitrates and admitted to ICU. No thienopyridines or heparin were given because of the likely need for pericardiocentesis (and no atherosclerosis was actually seen).

The next morning the patient became more tachycardic and uncomfortable. A transthoracic echocardiography showed mild LV dysfunction and about $2 \mathrm{~cm}$ of pericardial effusion. Signs of tamponade were present both clinically (tachycardia, pulsus paradoxus) and echocardiographically (swinging heart motion, right atrial collapse and dilated inferior vena cava), so pericardiocentesis was immediately performed. Three hundred milliliter of frank blood were drained, restoring hemodynamics. An urgent computer assisted tomography (CT) scan ruled out aortic dissection and ventricular pseudoaneurysm. The pericardial drain was removed the next day after exclusion of residual effusion by echo. Two days later however, he again developed chest pain, tachycardia and hypotension. A new transthoracic echocardiography showed reoccurrence of pericardial effusion. Pericardiocentesis was performed, again draining bloody fluid. From then on, recovery was uneventful. Repeated echocardiograms showed no recurrence of pericardial fluid. Cardiac magnetic resonance imaging (cMRI) revealed transmural infarction of the inferoposterior LV wall with central profound necrosis. Adjacent to this necrotic LV zone, an intrapericardial thrombus was seen, but no free fluid.

After a week he could leave the hospital in good clinical condition. Follow-up cMRI showed normal evolution of a transmural infarction with scar formation in the inferolateral wall.

Early on, an underlying connective tissue disease was suspected. Deeper anamnesis revealed a tendency of easy bruising. His son was born 4 years earlier with bilateral clubfeet. Clinical examination showed thin and translucent skin, a few atrophic scars (cigarette paper scars), micrognatia, short thumbs and a broad, short uvula. Further vascular imaging by means of CT scans, revealed a small aneurysm of the left vertebral artery, dissection of the right external iliac artery and a dissection with $19 \mathrm{~mm}$ aneurysm in the left external iliac artery (Figure 2).

The patient was referred for genetic testing, which revealed mutation of the type III procollagen gene (COL3A1). The diagnosis of Ehler-Danlos type 4 was therewith confirmed.

The patient was prescribed Celiprolol $200 \mathrm{mg}$ daily (which has shown to reduce vascular events in type 4 EDS $)^{2}$ and refrains from intensive or contact sports. He survives, free from any major events, for over 6 years now. 


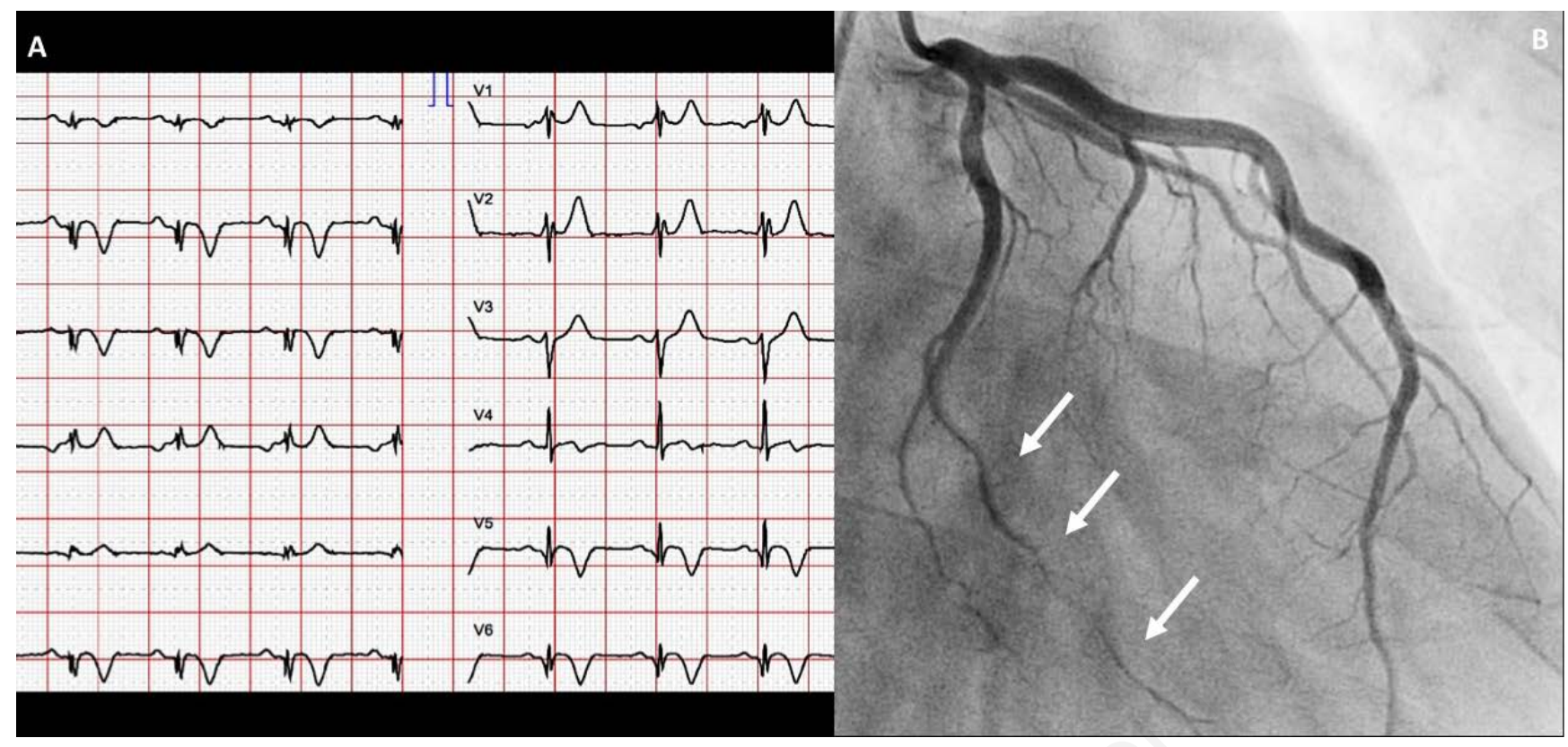

Figure 1. (A) 12-lead ECG showing $Q$ waves, deep negative $T$ waves end mild residual ST-segment elevation in the inferior and lateral leads; (B) still frame from the coronary angiography of the left coronary artery. White arrows show the dissected branch with diffuse loss of lumen, consistent with type 2 SCAD.

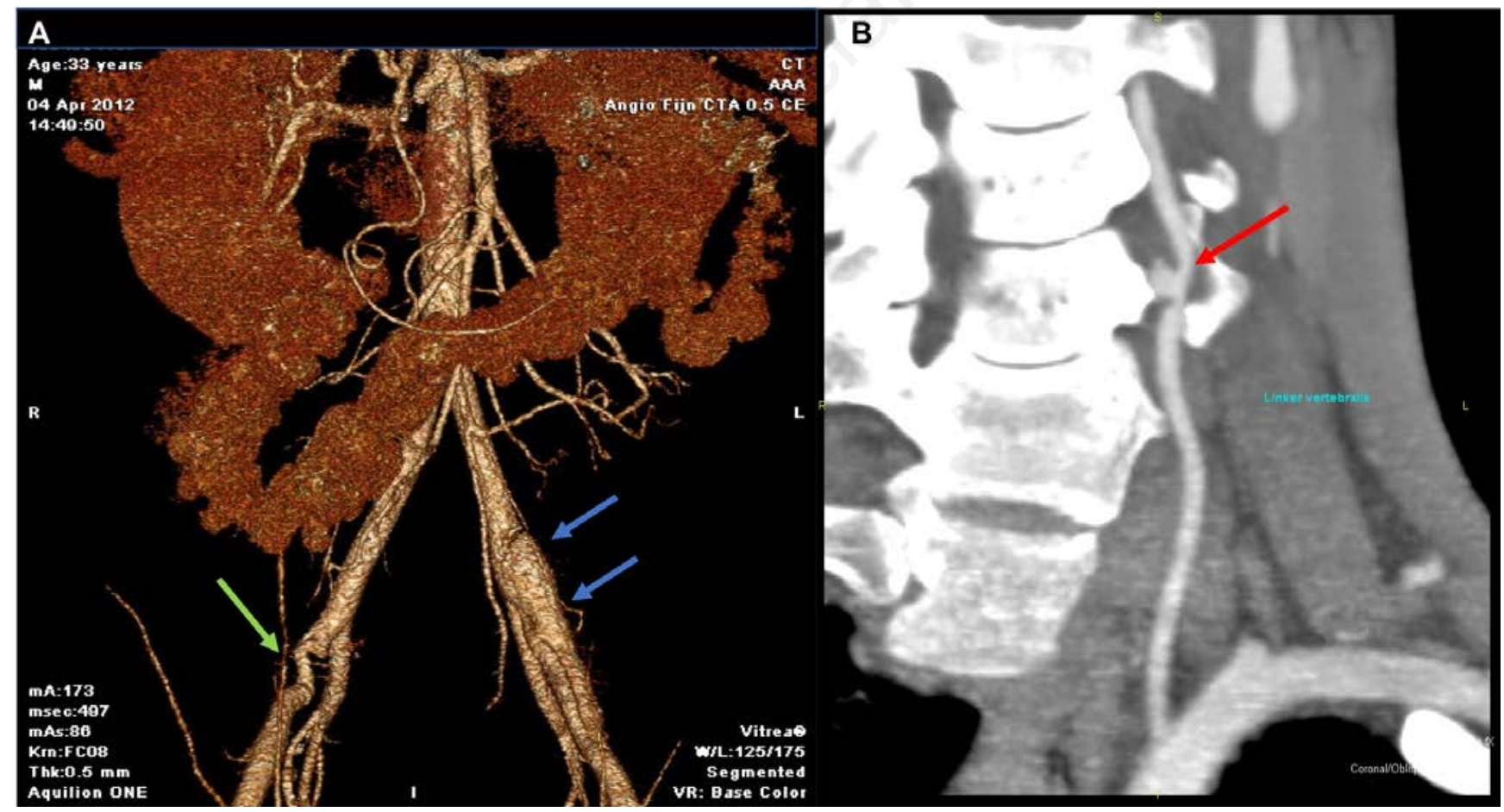

Figure 2. (A) 3D reconstruction of CT angiography of the abdominal arteries. Dissection and narrowing of the right external iliac artery (EIA, green arrow) and dissection with aneurysm of the left EIA (blue arrows). (B) maximum intensity projection of the smaller left vertebral artery, showing a small aneurysm. 


\section{Discussion}

\section{Spontaneous coronary artery dissection}

In the general population, spontaneous coronary artery dissection $(\mathrm{SCAD})^{3,4}$ is a rare cause of acute coronary syndrome, representing about 1-4 \%o of the cases. Most cases are seen in female patients $(90 \%)$. In a population of young women, presenting with ACS, however, SCAD accounts for up to $20 \%$ of cases.

The underlying mechanism of non-atherosclerotic spontaneous coronary artery dissection is not fully understood. In absence of trauma, the diagnosis of SCAD is made in most patients at the time of coronary angiography. In patients for whom the diagnosis is considered but not secured with coronary angiography, intracoronary imaging with optical coherence tomography or intravascular ultrasound may be helpful.

In most spontaneous coronary artery dissection patients, conservative therapy with nitrates and (at least) aspirin is the preferred strategy once the diagnosis is secured. The use of anticoagulants or dual antiplatelet therapy remains an area of discussion. In patients with persistent ST-elevation, cardiogenic shock, malignant arrhythmia and/or persistent major coronary artery obstruction, revascularization should be attempted by PCI or CABG. The sole presence of chest pain may be due to the arterial dissection itself and only warrants revascularization attempts when progression of ischaemia is objectivated.

Underlying causes of SCAD should be considered in all cases. This condition is seen in healthy young women, but also in the early postpartum, in patients with vasculitis (such as polyarteritis nodosa and lupus), blood vessel conditions as fibromuscular dysplasia (FMD), induced by cocaine use, by extreme exercise and also in connective tissue disorders (vascular Ehlers-Danlos syndrome and Loeys-Dietz syndrome).

Male patients (even though very rare) seem to have a higher prevalence of underlying FMD or connective tissue disorders.

Pericardial effusion and tamponade are not typically seen. ${ }^{5}$ When tamponade occurs, vascular or myocardial perforation or rupture should be suspected. Prompt drainage of the pericardium, as well as urgent imaging and cardiosurgical consult should be carried out.

\section{Vascular Ehlers-Danlos syndrome}

Vascular Ehlers-Danlos syndrome (VEDS) $)^{6,7}$ is a rare (1/50.000-1/200.000) inherited connective-tissue disorder which results from pathogenic variants in COL3A1. This gene encodes for the chains of type III procollagen, a major protein in vessel walls and hollow organs.

This causes profound vascular (both venous and arterial) and gastrointestinal fragility, leading to spontaneous rupture of the large arteries or large veins, uterus, or bowel. Any trauma or manipulation (including catheter placement, tourniquet application or puncture) can lead to rupture or dissection. Bleeding can be extremely difficult to control and is therefore potentially lifethreatening.
Our patient was previously undiagnosed with this condition. Strenuous physical exercise was judged to be the trigger for the occurrence of isolated SCAD. The infarction that ensued, most likely lead to covered myocardial perforation (or coronary artery branch rupture?), presenting as recurrent cardiac tamponade. Luckily, the invasive procedures performed, did not cause lifethreatening complications in our case.

\section{Conclusions}

Important messages for all physicians implicated in the care of patients with acute chest pain should be aware of the issues highlighted by this case:

i) SCAD is a distinct cause of acute coronary syndrome, typically presenting in young healthy women, possibly present in both sexes and patients of all ages. The diagnosis can only be made by coronary angiography (and sometimes intracoronary imaging). Modern coronary CT scan imaging may very well be able to be equally useful in these cases.

ii) In men presenting with $\mathrm{SCAD}$, a high degree of suspicion should arise for the presence of underlying vascular/ connective tissue disease.

iii) Patients with known or suspected VEDS are at very high risk of vascular (as well as intestinal or uterine) rupture. They should be managed with the greatest of care, avoiding punctures or catheter manipulations whenever possible. Whenever possible, referral to or at least consultation of a center with expertise in this rare condition is recommended.

\section{References}

1. Saw J, Huphries K. Spontaneous coronary artery dissection. Clinical outcomes and risk of recurrence. J Am Coll Cardiol 2017:70;1148-58.

2. Ong K, Perdu J, De Backer J, et al. Effect of celiprolol on prevention of cardiovascular events in vascular Ehlers-Danlos syndrome: a prospective randomised, open, blinded-endpoints trial. Lancet 2010;376:1476-84.

3. Nayes S, Kim E, Saw J, et al. Spontaneous coronary artery dissection: current state of the science. Circulation 2018;137:e523-57.

4. Tweet M, Hayes S. Clinical features, management and prognosis of spontaneous coronary artery dissection. Circulation 2012;126:579-88.

5. Goh A, Lundstrom R. Spontaneous coronary artery dissection with cardiac tamponade. Tex Heart Int J 2015;42:479-82.

6. Wiesmann T, Castori M, Malfait F, Wulf H. Recommendations for anesthesia and perioperative management in patients with Ehlers-Danlos syndrome(s). Orphanet J Rare Dis 2014;9:109.

7. De Paepe A, Malfait F. The Ehlers-Danlos syndrome, a disorder with many faces. Clin Genet 2012:82:1-11. 